\title{
Low meningitis-incidence in primary spontaneous compared to secondary cerebrospinal fluid rhinorrhoea*
}

\author{
Sophia C. Poletti-Muringaseril', Kaspar Rufibach², Christian Ruef3, \\ David Holzmann', Michael B. Soyka' \\ Department of Otolaryngology Head and Neck Surgery, University Hospital Zurich, Zurich, Switzerland \\ Division of Biostatistics, Institute for Social and Preventive Medicine, University of Zurich, Zurich, Switzerland \\ Department of Infectious Diseases and Hospital Epidemiology, University Hospital Zurich, Zurich, Switzerland
}

Rhinology 50: 73-79, 2012 DOI: 10.4193/Rhino11.124 *Received for publication: June 14, 2011

accepted: November 11, 2011

\section{Summary}

Objective: Spontaneous cerebrospinal fluid (CSF) rhinorrhoea is a separate clinical entity with elevated intracranial pressure as its most probable pathophysiological mechanism. Having the clinical impression of distinct courses of diseases in primary spontaneous (PS) compared to secondary CSF rhinorrhoea, our objective was to identify whether the two forms differ in the duration of CSF rhinorrhoea and the incidence of meningitis.

Methods: Chart review performed on all patients referred with a CSF leak to our tertiary-care medical center over a 20-year period from 1990 to 2010.

Results: In total, 58 cases suffering from CSF rhinorrhoea could be included. The aetiology was primary spontaneous in $23(40 \%)$ and secondary in 35 (60\%) patients. The duration of CSF rhinorrhoea was notably longer in patients with PS CSF rhinorrhoea. Moreover, we could show a significantly lower incidence of meningitis with PS CSF rhinorrhoea compared to the secondary group (annual incidence of 0.12 vs. 1.22 episodes).

Conclusion: A significantly lower incidence and delayed onset of meningitis in patients suffering from PS CSF rhinorrhoea could be explained by an elevated intracranial pressure that hinders the ascension of bacteria. The closure of a leak in secondary CSF fistula seems more urgent than in PS CSF fistulas.

Key words: cerebrospinal fluid rhinorrhoea, spontaneous, cerebrospinal fluid leak, meningitis, primary, secondary

\section{Introduction}

Communication of the paranasal sinuses or nasal cavity with the subarachnoid space leads to cerebrospinal fluid (CSF) rhinorrhoea. Furthermore, this communication allows infections to enter the subarachnoid space and cause potentially life-threatening meningitis. There are different classifications of CSF rhinorrhoea used in the literature. While Har-EI ${ }^{(1)}$, and Adoga ${ }^{(2)}$ used the classification of traumatic and non-traumatic leaks, Giannetti et al., ${ }^{(3)}$ distinguished between primary and secondary CSF rhinorrhoea in their latest publication. In accordance with the latest publication, CSF leaks due to trauma, tumour, surgery and previous radiation therapy is referred to as secondary CSF rhinorrhoea (SEC CSF rhinorrhoea). A CSF- leak that is not related to any of these events is called primary spontaneous CSF rhinorrhoea (PS CSF rhinorrhoea). Since the pathophysiological mechanisms are not yet fully understood, PS CSF rhinorrhoea poses increasing diagnostic and therapeutic challenges for any ENT specialist.

Up to the late 70s, patients with spontaneous leaks were described as having normal CSF pressure ${ }^{(4,5)}$, whereas today PS CSF rhinorrhoea is postulated to be accompanied by elevated intracranial pressure (ICP).

Increased ICP leads to bone erosion through continuous pulsatile pressure on anatomically weak sites, mainly within the frontal skull base ${ }^{(6)}$. A disproportionately high number of patients with excessive sinus pneumatisation could be found among those 
who suffer from PS CSF rhinorrhoea ${ }^{(7)}$. Due to the perception of elevated ICP accompanied by symptoms like headache, pulsatile tinnitus and balance problems as well as demographical signs such as obesity, middle-age and female sex, PS CSF rhinorrhoea was even suggested to present a subgroup of benign intracranial hypertension $(\mathrm{BIH})^{(8,9)}$. The clinical association between spontaneous CSF-leak and the empty sella syndrome as an indicator of raised intracranial pressure suggests a common pathophysiology ${ }^{(10,11)}$. Anatomically weakened regions such as the ethmoid roof, cribriform plate and pneumatised lateral recess of the sphenoid sinus seem to relent first ${ }^{(6,11-13)}$.

So far arachnoid granulations have been known to cause temporal bone erosions that presented with CSF otorrhea ${ }^{(14,15)}$. Recent publications, however, disclosed radiographical signs of empty or partial empty sella and typical demographical signs as described above ${ }^{(16)}$, which assumes the presence of elevated intracranial pressure even with spontaneous CSF- otorrhea.

The risk of acquiring meningitis in active CSF fistula is being estimated between $10 \%$ and $36.5 \%$, whereas SEC CSF rhinorrhoea due to trauma has the highest prevalence ${ }^{(17-19)}$. Up until now, an early endoscopic closure is recommended to reduce the risk of meningitis ${ }^{(20-22)}$. Antibiotic prophylaxis has not shown any significant reduction in the risk of meningitis in SEC CSF rhinorrhoea ${ }^{(23,24)}$.

According to the observations and experiences of our ENT department in managing patients with CSF leaks, we had the impression of a prolonged duration of CSF rhinorrhoea and still a lower incidence of meningitis in patients with PS CSF rhinorrhoea in comparison to the secondary group. Thus, the objective of our study was to substantiate our hypothesis and verify whether the two groups differ in the incidence of meningitis and duration of CSF rhinorrhoea. In this context, it would also be of great interest whether an early endoscopic closure is likewise needed in PS CSF leakage as it is in the secondary type.

\section{Materials and methods}

Study design

The study protocol was approved by the local ethics committee and review board. It was conducted in accordance with the latest version of the Helsinki declarations.

A retrospective data analysis was performed on patients diagnosed with CSF fistula and admitted to the ENT department of the University Hospital of Zurich between 1990 and 2010. The CSF leak was identified by a positive beta-2-transferrin test and/ or a bone dehiscence of the skull base on CT scan together with the symptom of clear rhinorrhoea.

\section{Patients}

Patients with a history of rhinorrhoea due to trauma, tumour, surgery or previous radiation therapy of the head were designa- ted as the group of secondary CSF rhinorrhoea. A proven CSFleak that was not related to any of these events was categorised as primary spontaneous CSF rhinorrhoea. Exclusion criteria were an incomplete data set, the uncertainty of diagnosis (e.g. inadequately documented and/ or untreated head trauma in the past which made an allocation to primary or secondary CSF rhinorrhoea difficult), meningitis prior to the history of CSF rhinorrhoea and lack of follow up.

Different quantities were recorded from the patient's history, including gender, age, beginning and duration of rhinorrhoea, date of meningitis and/or operation, location of defect, aetiology (type) of rhinorrhoea, antibiotic treatment and duration of follow up.

We compared the duration of CSF rhinorrhoea between both groups using the endpoint 'Time to Meningitis' (TTM). It was defined as the time period between the first appearance of CSF rhinorrhoea (from patients' history or directly visible clear rhinorrhoea) and the date of the first symptoms of meningitis, which were diagnosed later on by lumbar puncture. If the patient underwent surgery before any event of meningitis, the operation date was used to assess this period. Two competing events (meningitis and operation) were limiting the duration of CSF rhinorrhoea, so a 'competing risk' analysis was required rather than the commonly used 'Kaplan-Meier' estimates to compute the duration of CSF rhinorrhoea. Patients could not simply be censored on the operation date because being operated altered the chances of experiencing meningitis. Hence, having an operation was a competing risk event ${ }^{(25,26)}$. The probability of each considered event (meningitis or operation) was estimated at $3,6,12,24$ and 48 months after appearance of the CSF rhinorrhoea, including the 95\% confidence interval as shown in Table 1.

\section{Statistical analysis}

To estimate the incidence rates of meningitis in both groups, we used maximum likelihood estimates from a Poisson model, where the number of meningitis events was divided by the total observation time given in person years. Confidence intervals for the log-rate were computed and back- transformed to the original scale ${ }^{(27)}$.

All analyses were performed using R software (R Development Core Team, 2010) ${ }^{(28)}$. The cumulative incidence plots shown are generated using the R package cmprsk (version 2.1-7) ${ }^{(29)}$. A significance level of 0.05 was assumed for all analyses and all confidence intervals were computed at a confidence level of $95 \%$.

\section{Results}

Between 1990 and 2010, sixty-seven patients were identified with the diagnosis of CSF fistula using our electronic patient database. Seven patients were excluded from the study because of incomplete data. Due to missing information about previous trauma in their history, the assignment to a certain group of CSF rhinorrhoea was not possible. Furthermore, the 
beginning of CSF rhinorrhoea could not be reproduced from further patients' medical data. Two patients had a history of meningitis prior to the onset of CSF rhinorrhoea. These patients might have had an unperceived CSF rhinorrhoea but due to the fact that we could not prove an active CSF rhinorrhoea and also could not define the beginning, we consequently excluded these patients as well.

Of the remaining 58 patients, CSF rhinorrhoea was diagnosed as primary spontaneous in $23(40 \%)$ and secondary in $35(60 \%)$ patients. Median age was 52 (range 30 - 69 years) in primary and 46 (range 4 - 86) in secondary CSF rhinorrhoea. The causes of secondary CSF leaks were postoperative $(10 / 58=17 \%)$, posttraumatic $(17,29 \%)$ and due to tumour destruction or its therapy $(8,14 \%)$ in the skull base region. Table 2 shows age and gender distribution, aetiology and site of the CSF leak as well as the application of antibiotics within the two groups.

Among spontaneous CSF leaks, the defect was mostly located in the ethmoid roof $(16,70 \%)$ whereas secondary CSF leaks were found in the sphenoid sinus $(14,40 \%)$ and ethmoid roof $(13,37 \%)$.

Of patients with PS CSF rhinorrhoea, 22\% (5) received prophylactic antibiotics to prevent meningitis, whereas $54 \%$ (19) of patients from the secondary group were treated with miscellaneous antibiotics during an active CSF rhinorrhoea. Ten (29\%) of the cases with SEC CSF rhinorrhoea developed complications of meningitis prior to surgery, whereas only 4 (17\%) patients with primary CSF leak suffered from meningitis. The overall rate of meningitis can be read off the cumulative incidence estimates in Figure 1 at a time point later than the last event and are added to Table 1.

Using the Poisson model, we can conclude from the nonoverlapping confidence intervals given in Table 3, that there is a significant difference in the estimated incidence rates of meningitis between primary and secondary CSF rhinorrhoea. The annual incidence of developing meningitis due to PS CSF leaks is $0.12(95 \% \mathrm{CI}[0.05,0.32])$ episodes per year and even 1.22 $(95 \% \mathrm{Cl}[0.66,2.27])$ episodes per year due to SEC CSF rhinorrhoea (Table 3).

We were able to demonstrate differences in the duration of CSF rhinorrhoea between these two groups, but a statistical significance could not be shown. Patients with PS CSF fistula had a median time of persisting CSF rhinorrhoea of 0.9 years before they underwent surgical treatment to close CSF leakage. Meanwhile those suffering from SEC CSF leakage had a shorter duration of CSF rhinorrhoea after an early endoscopic closure with a median time of 0.1 year (Figure 1, Table 4). Even though endoscopic closure was performed much earlier in SEC CSF leaks, the observed overall rate of meningitis was still higher in a shorter period within these patients ( $29 \%$ versus $17 \%$ ).

\section{Discussion}

Confirming the diagnosis, knowing the aetiology and detecting the site of defect are all arbitrative for optimal management of CSF leaks. Due to the confusing terminology and the increasing importance of comparing results in studies of CSF rhinorrhoea, an internationally accepted classification is much needed. In accordance to the classification of Giannetti et al., we divided our patients into a primary and a secondary group ${ }^{(3)}$. We believe that the aetiologies unified as secondary CSF rhinorrhoea in our study have a common pathophysiological mechanism through direct skull base destruction, whereas PS CSF rhinorrhoea leads through intracranial hypertension to CSF fistula $(9,10)$.

Increasing attention is being paid to CSF leaks of spontaneous origin where elevated intracranial pressure seems to be the pathophysiological cause of bone erosion at predilection sites ${ }^{(6)}$. Our study shows similar findings as shown in the literature with $70 \%$ of primary spontaneous CSF leaks located at the ethmoid roof (and only $26 \%$ at the sphenoid sinus) and $80 \%$ of secondary CSF leaks found equally either at the sphenoid sinus or the ethmoid roof ${ }^{(22)}$.

Bacterial meningitis as a potential complication of active CSF leakage plays a very important role for the management of patients with proven CSF rhinorrhoea. The overall risk of suffering from meningitis in untreated CSF leaks is described in the literature as $10 \%$ to $36 \%(17,18,21,30)$.

Daudia et al., found an overall risk of developing meningitis in $32 \%$ with secondary CSF leak only due to trauma and a risk of $10 \%$ with PS CSF rhinorrhoea ${ }^{(31)}$. Our series show similar findings with an observed overall incidence of $17 \%$ for PS CSF rhinorrhoea and $29 \%$ for all SEC CSF rhinorrhoea, but our series revealed a significant difference in the incidence of meningitis between these two groups especially with a lower incidence in patients with primary spontaneous than in those with secondary CSF leakage. According to the etilogy, Daudia et al., divided SEC CSF rhinorrhoea into subgroups with overall risks computed separately, whereas in our study irrespective of the aetiology, SEC CSF rhinorrhoea was combined in one group.

There are several studies showing an association between PS CSF rhinorrhoea and elevated intracranial pressure (ICP). Schlosser et al., showed that 10 out of 16 patients with PS CSF rhinorrhoea underwent postoperative lumber puncture with CSF pressure measurement. All patients had elevated ICP and even 15 patients showed complete (10) or partial (5) empty sella syndrome as a radiographical sign of elevated ICP ${ }^{(6,10)}$. However, there are still controversies about the reliability of the lumber opening pressure during lumbar puncture and its association with the intracranial pressure ${ }^{(32,33)}$. Likewise, Seth et al., showed that the diagnosis of benign intracranial hypertension in patients with PS CSF rhinorrhoea is associated with the need 
Table 1. Estimates of Incidences for each event (meningitis/ operation) at given time points with $95 \%$ confidence interval and overall cumulative incidence of meningitis.

\begin{tabular}{|c|c|c|c|c|c|c|c|}
\hline & & 3 months & 6 months & 12 months & 24 months & 48 months & $\begin{array}{l}\text { Overall Cumulative } \\
\text { Incidence }\end{array}$ \\
\hline$\frac{n}{2}$ & $\begin{array}{l}\text { Primary CSF } \\
\text { rhinorrhoea }\end{array}$ & $\begin{array}{c}0.04 \\
{[0.00,0.13]}\end{array}$ & $\begin{array}{c}0.04 \\
{[0.00,0.13]}\end{array}$ & $\begin{array}{c}0.09 \\
{[0.00,0.21]}\end{array}$ & $\begin{array}{c}0.13 \\
{[0.00,0.28]}\end{array}$ & $\begin{array}{c}0.13 \\
{[0.00,0.28]}\end{array}$ & $\begin{array}{c}17 \% \\
{[0 \%, 35 \%]}\end{array}$ \\
\hline$\frac{\frac{5}{0}}{2}$ & $\begin{array}{l}\text { Secondary CSF } \\
\text { rhinorrhoea }\end{array}$ & $\begin{array}{c}0.17 \\
{[0.04,0.30]}\end{array}$ & $\begin{array}{c}0.20 \\
{[0.06,0.34]}\end{array}$ & $\begin{array}{c}0.26 \\
{[0.11,0.41]}\end{array}$ & $\begin{array}{c}0.29 \\
{[0.13,0.45]}\end{array}$ & & $\begin{array}{c}29 \% \\
{[13 \%, 45 \%]}\end{array}$ \\
\hline 흘 & $\begin{array}{l}\text { Primary CSF } \\
\text { rhinorrhea }\end{array}$ & $\begin{array}{c}0.17 \\
{[0.02,0.33]}\end{array}$ & $\begin{array}{c}0.39 \\
{[0.19,0.60]}\end{array}$ & $\begin{array}{c}0.57 \\
{[0.35,0.78]}\end{array}$ & $\begin{array}{c}0.65 \\
{[0.45,0.86]}\end{array}$ & $\begin{array}{c}0.78 \\
{[0.60,0.97]}\end{array}$ & \\
\hline ○ั & $\begin{array}{c}\text { Secondary CSF } \\
\text { rhinorrhoea }\end{array}$ & $\begin{array}{c}0.63 \\
{[0.46,0.79]}\end{array}$ & $\begin{array}{c}0.66 \\
{[0.52,0.82]}\end{array}$ & $\begin{array}{c}0.69 \\
{[0.52,0.85]}\end{array}$ & $\begin{array}{c}0.69 \\
{[0.52,0.85]}\end{array}$ & & \\
\hline
\end{tabular}

Table 2. Descriptive statistics: Comparing Primary and Secondary CSF Rhinorrhoea according to gender, aetiology, antibiotics and location of leak.

\begin{tabular}{|c|c|c|c|c|c|c|c|}
\hline Variable & Levels & n primary & $\%$ primary & n secondary & $\%$ secondary & $\mathrm{n}$ all & $\%$ all \\
\hline \multirow[t]{3}{*}{ Sex } & $\mathrm{F}$ & 15 & 65.2 & 19 & 54.3 & 34 & 58.6 \\
\hline & M & 8 & 34.8 & 16 & 45.7 & 24 & 41.4 \\
\hline & All & 23 & 100.0 & 35 & 100.0 & 58 & 100.0 \\
\hline \multirow[t]{5}{*}{ Etiology } & Postop & - & - & 10 & 28.6 & 10 & 17.2 \\
\hline & Primary & 23 & 100 & - & - & 23 & 39.7 \\
\hline & Trauma & - & - & 17 & 48.6 & 17 & 29.3 \\
\hline & Tumour & - & - & 8 & 22.9 & 8 & 13.8 \\
\hline & All & 23 & 100 & 35 & 100 & 58 & 100.0 \\
\hline \multirow[t]{3}{*}{ Antibiotics } & No & 18 & 78.3 & 16 & 45.7 & 34 & 58.6 \\
\hline & Yes & 5 & 21.7 & 19 & 54.3 & 24 & 41.4 \\
\hline & All & 23 & 100.0 & 35 & 100.0 & 58 & 100.0 \\
\hline \multirow[t]{6}{*}{ Location } & Ethmoid & 16 & 69.6 & 13 & 37.1 & 29 & 50 \\
\hline & Frontal sinus & 0 & 0.0 & 5 & 14.3 & 5 & 8.6 \\
\hline & $\begin{array}{l}\text { Frontal/ } \\
\text { ethmoid }\end{array}$ & 1 & 4.3 & 1 & 2.9 & 2 & 3.4 \\
\hline & Sphenoid & 6 & 26.1 & 14 & 40.0 & 20 & 34.5 \\
\hline & $\begin{array}{l}\text { Sphenoid / } \\
\text { ethmoid }\end{array}$ & 0 & 0.0 & 2 & 5.7 & 2 & 3.4 \\
\hline & All & 23 & 100 & 35 & 100.0 & 58 & 100.0 \\
\hline
\end{tabular}

n primary / \% primary: number / percentage of patients with Primary Spontaneous CSF Rhinorrhoea n secondary / \% secondary: number / percentage of patients with Secondary CSF Rhinorrhoea $\mathrm{n}$ all / \% all : number / percentage of all patients with CSF Rhinorrhoea 
Table 3. Annual incidence of meningitis in patients with Primary and Secondary CSF Rhinorrhoea.

\begin{tabular}{lcccc} 
& Patients with meningitis & $\begin{array}{c}\text { Total observation time } \\
\text { in person year }\end{array}$ & $\begin{array}{c}\text { Annual incidence of } \\
\text { meningitis }\end{array}$ & $\begin{array}{c}95 \% \text { confidence interval } \\
\text { (CI) }\end{array}$ \\
\hline $\begin{array}{l}\text { Primary CSF- rhinorrhea } \\
\text { Secondary CSF- rhinorrhea }\end{array}$ & 4 & 33.2 & 0.120 & {$[0.045,0.321]$} \\
\hline All & 10 & 8.2 & 1.222 & {$[0.657,2.270]$} \\
\hline
\end{tabular}

Table 4. Median duration of CSF Rhinorrhoea till surgery, in years.

0.5-Quantile

(years)

Primary CSF rhinorrhea, surgery

0.9

Secondary CSF rhinorrhea, surgery

0.1

of further treatments after endoscopic repair ${ }^{(34)}$. In consideration of our findings the important question came up, if elevated intracranial pressure might be the reason for a significantly lower incidence of meningitis by hindering ascension of bacterial meningitis through a high-pressure leak in patients with PS CSF rhinorrhoea.

Even though primary and secondary CSF rhinorrhoea do not show statistically significant differences in the duration of CSF rhinorrhoea, we could clearly show a tendency in both groups (Figure 1). Our series demonstrates that although patients with SEC CSF rhinorrhoea have a shorter duration of rhinorrhoea and have undergone earlier endoscopic closure, the risk of meningitis during active CSF rhinorrhoea was still higher than in patients with PS CSF leaks. Having a significantly lower incidence of meningitis and a comparatively lower risk of meningitis during active CSF rhinorrhoea, our study indicates that an elective planning of the operative procedure instead of an immediate surgical closure of spontaneous CSF leak might be possible. Considering the fact that all patients still have the potential to suffer from life- threatening meningitis, there is no doubt about the necessity of surgical closure. It is more of a question of urgency, but a 'safe' interval can hardly be defined.

Between 1993 and 1997, 5 (22\%) patients with PS CSF rhinorrhoea received prophylactic antibiotics to prevent ascending meningitis. Nineteen (54\%) cases with SEC CSF rhinorrhoea, mostly traumatic $(8,23 \%)$ or postoperative $(8,23 \%)$ cases, were treated with different antibiotics. Detailed information about type of antibiotic and duration of treatment could not be assessed conclusively from patient's history. Even though fewer patients with PS CSF rhinorrhoea received antibiotics, the observed overall incidence of meningitis was still lower than with SEC CSF rhinorrhoea (17\% versus 29\%). Despite missing evidence, from our findings we presume no relevant benefit from prophylactic antibiotics in PS CSF rhinorrhoea. Therefore, like in SEC CSF rhinorrhoea, we cannot recommend prophylactic antibiotics to prevent meningitis in primary spontaneous CSF leaks $(23,24,35,36)$.

In spite of all findings, we have to state that the weakness of this retrospective study seems to be the uncommon clinical picture of PS CSF rhinorrhoea with a comparatively small number of patients, where data was collected over a period of 20 years. Thus different time dependent changes in the health care system could not be assessed. Due to the need of excluding nine patients from a small patient collective, this bias might potentially lead to an 'optimistic' study outcome, but exclusion seemed rather random. Furthermore, relying on patient's history, like in our study in terms of the beginning of rhinorrhoea, also makes a study prone to bias. Although a coherency of intracranial hypertension and hindered ascension of bacteria cannot be assessed conclusively by this retrospective study, we are still able to provide evidence for a decreased rate of meningitis in PS CSF rhinorrhoea and the need of an adapted therapeutic approach in those two distinct disease entities. Furthermore our study justifies further projects on elucidating the pathophysiological mechanisms of PS CSF rhinorrhoea. 


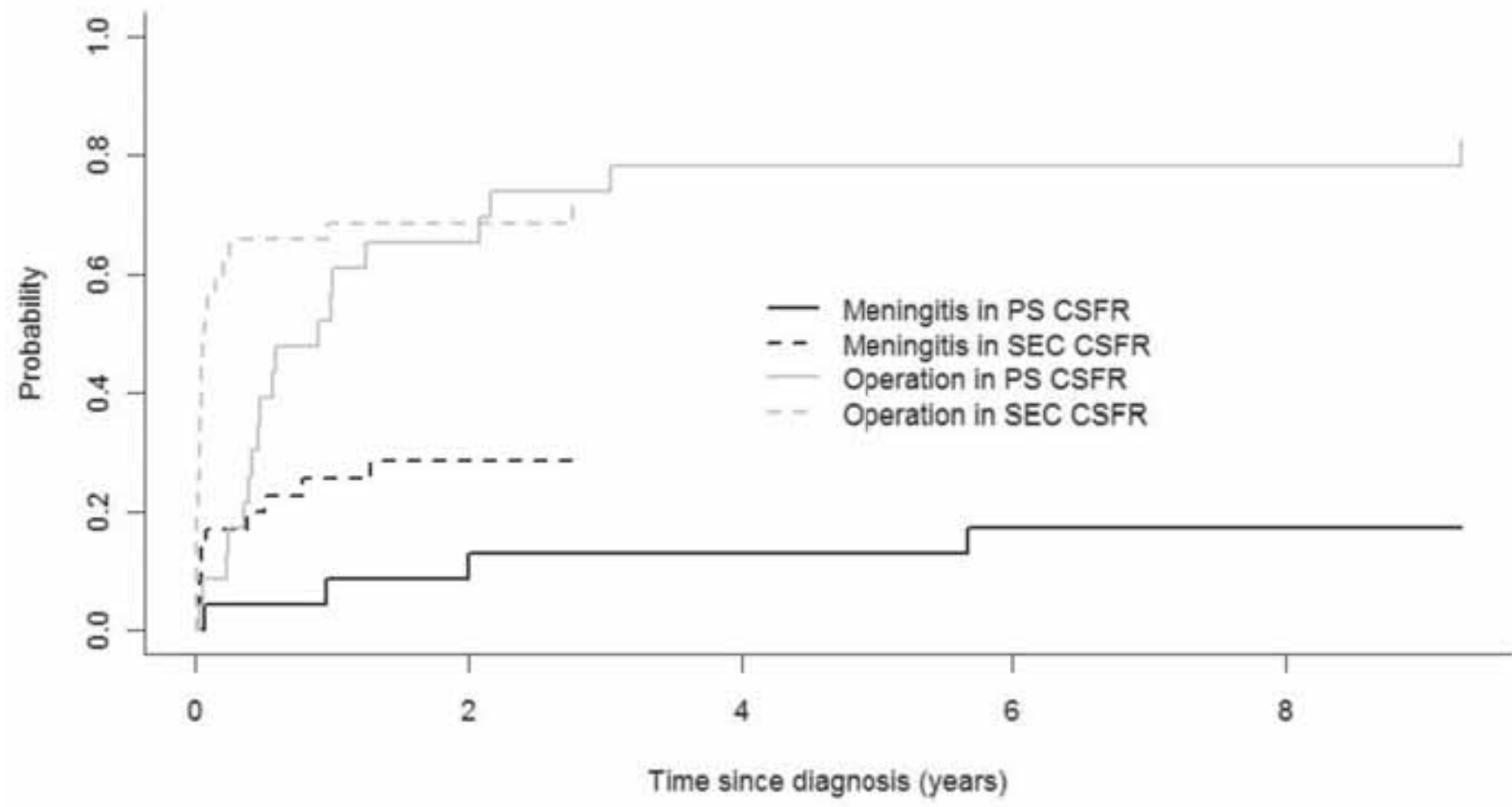

Figure 1. Cumulative incidence for the events "meningitis" and "operation" for all patients, by groups.

\section{Conclusion}

Even though the complete pathophysiological mechanism of primary spontaneous CSF rhinorrhoea is not yet understood, this study reveals important new findings in a unique disease. We found a significantly lower incidence and a delayed onset of meningitis in primary spontaneous CSF rhinorrhoea compared to the secondary form. A possible explanation might be elevated intracranial pressure presenting as a high-pressure leak, which hinders the ascension of bacteria in patients with primary spontaneous CSF rhinorrhoea. If CSF rhinorrhoea is suspected, its diagnosis must be pursued aggressively. To prevent ascending meningitis sealing of the CSF fistula must be performed even sooner in patients with secondary CSF fistula.

\section{Authorship contribution}

SP has analysed all data and written the manuscript; KR has performed statistical computing and revised the manuscript; CR has revised the manuscript with special considerations of an infectious disease specialist; $\mathrm{DH}$ has planned the study and revised the manuscript; MS has analysed the data and written the manuscript.

\section{Conflict of interest}

The authors state that there is no conflict of interest or founding of the project.

\section{References}

1. Har-El G. What is "spontaneous" cerebrospinal fluid rhinorrhoea? Classification of cerebrospinal fluid leaks. Ann Otol Rhinol Laryngol. 1999; 108: 323-326.

2. Adoga AA. Cerebrospinal fluid rhinorrhoea-an overview. Niger J Med. 2009; 18: 244-249.

3. Giannetti AV, de Morais Silva Santiago AP, Becker HM, Guimaraes RE. Comparative study between primary spontaneous cerebrospinal fluid fistula and late traumatic fistula. Otolaryngol Head Neck Surg. 2011; 144: 463-468

4. Ommaya AK, Di Chiro G, Baldwin M,
Pennybacker JB. Non-traumatic cerebrospinal fluid rhinorrhoea. J Neurol Neurosurg Psychiatry. 1968; 31: 214-225.

5. Hubbard JL, McDonald TJ, Pearson BW, Laws ER, Jr. Spontaneous cerebrospinal fluid rhinorrhoea: evolving concepts in diagnosis and surgical management based on the Mayo Clinic experience from 1970 through 1981. Neurosurgery. 1985; 16: 314-321.

6. Schlosser RJ, Wilensky EM, Grady MS, Bolger WE. Elevated intracranial pressures in spontaneous cerebrospinal fluid leaks. Am J Rhinol. 2003; 17: 191-195.

7. Shetty PG, Shroff MM, Sahani DV, Kirtane
MV. Evaluation of high-resolution CT and MR cisternography in the diagnosis of cerebrospinal fluid fistula. AJNR Am J Neuroradiol. 1998; 19: 633-639.

8. Holzmann D, Wild C. Obesity as a risk factor for primary spontaneous rhinoliquorrhea. Arch Otolaryngol Head Neck Surg. 2003; 129: 324-326.

9. Schlosser RJ, Woodworth BA, Wilensky EM Grady MS, Bolger WE. Spontaneous cerebrospinal fluid leaks: a variant of benign intracranial hypertension. Ann Otol Rhinol Laryngol. 2006; 115: 495-500.

10. Schlosser RJ, Bolger WE, Spontaneous nasal cerebrospinal fluid leaks and empty 
sella syndrome: a clinical association. Am J Rhinol. 2003; 17: 91-96.

11. Schuknecht B, Simmen D, Briner HR, Holzmann D. Nontraumatic skull base defects with spontaneous CSF rhinorrhoea and arachnoid herniation: imaging findings and correlation with endoscopic sinus surgery in 27 patients. AJNR Am J Neuroradiol. 2008: 29: 542-549.

12. Clark D, Bullock P, Hui T, Firth J. Benign intracranial hypertension: a cause of CSF rhinorrhoea. J Neurol Neurosurg Psychiatry. 1994; 57: 847-849.

13. Schlosser RJ, Wilensky EM, Grady MS, Palmer JN, Kennedy DW, Bolger WE. Cerebrospinal fluid pressure monitoring after repair of cerebrospinal fluid leaks. Otolaryngol Head Neck Surg. 2004; 130 : 443-448.

14. Gacek RR. Arachnoid granulation cerebrospinal fluid otorrhea. Ann Otol Rhinol Laryngol. 1990; 99: 854-862.

15. Leonetti JP, Marzo S, Anderson D, Origitano T, Vukas DD. Spontaneous transtemporal CSF leakage: a study of 51 cases. Ear Nose Throat J. 2005; 84: 700, 2-4, 6

16. Goddard JC, Meyer T, Nguyen S, Lambert PR. New considerations in the cause of spontaneous cerebrospinal fluid otorrhea. Otol Neurotol. 2010; 31: 940-945.

17. Eljamel MS, Foy PM. Acute traumatic CSF fistulae: the risk of intracranial infection. $\mathrm{Br}$ J Neurosurg. 1990; 4: 381-385.

18. Bernal-Sprekelsen M, Bleda-Vazquez C, Carrau RL. Ascending meningitis secondary to traumatic cerebrospinal fluid leaks. Am J Rhinol. 2000; 14: 257-259.

19. McMains KC, Gross CW, Kountakis SE. Endoscopic management of cerebrospinal fluid rhinorrhoea. Laryngoscope. 2004: 114 : 1833-1837.

20. Mirza S, Thaper A, McClelland L, Jones NS. Sinonasal cerebrospinal fluid leaks: man- agement of 97 patients over 10 years. Laryngoscope. 2005; 115: 1774-1777.

21. Hegazy HM, Carrau RL, Snyderman $\mathrm{CH}$, Kassam A, Zweig J. Transnasal endoscopic repair of cerebrospinal fluid rhinorrhoea: a meta-analysis. Laryngoscope. 2000; 110 : 1166-1172.

22. Banks CA, Palmer JN, Chiu AG, O'Malley BW, Jr., Woodworth BA, Kennedy DW. Endoscopic closure of CSF rhinorrhoea: 193 cases over 21 years. Otolaryngol Head Neck Surg. 2009; 140: 826-833.

23. Moralee SJ. Should prophylactic antibiotics be used in the management of cerebrospinal fluid rhinorrhoea following endoscopic sinus surgery? A review of the literature. Clin Otolaryngol Allied Sci. 1995; 20: 100-102.

24. Ratilal B, Costa J, Sampaio C. Antibiotic prophylaxis for preventing meningitis in patients with basilar skull fractures. Cochrane Database Syst Rev. 2006: CD004884.

25. Schumacher M, Schulgen G. Methodik klinischer Studien. Springer, Berlin 2002.

26. Satagopan JM, Ben-Porat L, Berwick M, Robson M, Kutler D, Auerbach AD. A note on competing risks in survival data analysis. Br J Cancer. 2004: 91: 1229-1235.

27. Kirkwood BR, Sterne JAC. Essential medical statistics. 2nd ed. Malden, Mass.: Blackwell Science; 2003.

28. Team RDC, 3-900051-07-0 I. R A Language and Environment for Statistical Computing. R Foundation for Statistical Computing, Vienna, Austria. 2010.

29. Gray B. Subdistribution Analysis of Competing Risks. R package version 21-7 2006.

30. Bernal-Sprekelsen M, Alobid I, Mullol J, Trobat F, Tomas-Barberan M. Closure of cerebrospinal fluid leaks prevents ascending bacterial meningitis. Rhinology. 2005; 43: 277-281
31. Daudia A, Biswas D, Jones NS. Risk of meningitis with cerebrospinal fluid rhinorrhoea. Ann Otol Rhinol Laryngol. 2007; 116: 902905.

32. Gucer G, Viernstein L. Long-term intracranial pressure recording in the management of pseudotumor cerebri. J Neurosurg. 1978; 49: 256-263.

33. Friedman DI. Idiopathic intracranial hypertension with Dan and beyond: the 2010 Jacobson Lecture. J Neuroophthalmol. 2010; 30: 380-385.

34. Seth R, Rajasekaran K, 3rd, Luong A, Benninger MS, Batra PS. Spontaneous CSF leaks: factors predictive of additional interventions. Laryngoscope. 2010; 120: 21412146 .

35. Eljamel MS. Antibiotic prophylaxis in unrepaired CSF fistulae. Br J Neurosurg. 1993; 7: 501-505.

36. Villalobos T, Arango C, Kubilis P, Rathore M. Antibiotic prophylaxis after basilar skull fractures: a meta-analysis. Clin Infect Dis. 1998; 27: 364-369.

\section{David Holzmann}

University Hospital Zurich

Department Otolaryngology Head

and Neck Surgery

Frauenklinikstrasse 24

8091 Zurich

Switzerland

E-mail: david.holzmann@usz.ch

Tel: +41-44-255 1111

Fax: +41-44-255 4556 